\title{
Dust processing in the interstellar medium
}

\author{
Hiroyuki Hirashita* \\ Institute of Astronomy and Astrophysics, Academia Sinica, Taipei, Taiwan \\ E-mail: hirashitalasiaa.sinica.edu.tw
}

In considering the evolution of dust in the interstellar medium (ISM), there are two aspects to be considered: the dust mass and the dust properties. We review various dust formation and destruction processes that affect these two aspects. In particular, we focus on dust processing in the ISM: (i) grain growth by the accretion of gas-phase metals; (ii) grain growth by coagulation; (iii) shattering (grain disruption/fragmentation); and (iv) dust destruction by supernova shocks. These processes are strongly entangled and affect both the grain size distribution and the total dust mass. Therefore, in order to understand the evolution of dust over the entire galaxy evolution, we should consider not only the dust production and destruction by stellar sources, but also all those interstellar processes consistently. We put particular focus on the effects of those processes on the extinction curve.

The Life Cycle of Dust in the Universe: Observations, Theory, and Laboratory Experiments 18-22 November, 2013

Taipei, Taiwan

* Speaker. 


\section{Introduction}

One of the most important problems in astronomy and astrophysics is the origin and evolution of dust in the Universe. Indeed there are some major aspects of galaxy evolution significantly influenced by the dust content. Dust governs the absorption, scattering, and reemission of the stellar light, affecting the radiative transfer in the interstellar medium (ISM) [1-3]. Furthermore, the surfaces of dust grains are the main site for the formation of some molecular species, especially $\mathrm{H}_{2}[4,5]$. The chemical reactions occurring on dust surfaces could affect the evolution of the physical and chemical evolution of the ISM. Therefore, clarifying the origin and evolution of dust content is essential for revealing how galaxies have evolved in the Universe.

It is widely believed that the evolution of dust content in galaxies occurs through dust formation in stellar ejecta, dust destruction in supernovae (SN) remnants, and grain growth by the accretion of metals onto preexisting grains in molecular clouds [6-12]. The dust grains are also processed by shattering (disruption and/or fragmentation) $[13,14]$ and coagulation (sticking) [1517], although these processes do not change the total dust mass but only changes the grain size distribution, the importance of which will be described later. The first purpose of this review is to see at which stage of galaxy evolution each of the above processes dominates the dust evolution.

The grain size distribution is one of the most important aspects in dust properties. The grain size distribution (as a function of grain radius $a$ ), $n(a)$, is defined so that the number density of grains with radius between $a$ and $a+\mathrm{d} a$ is $n(a) \mathrm{d} a$. The extinction curve, which is the wavelength dependence of dust extinction (Section 2.2), depends on the grain size distribution as well as the grain compositions [e.g. 18-21]. The $\mathrm{H}_{2}$ formation rate on dust surfaces also depends on the grain size distribution [22]. Therefore, the grain size distribution is no less important than the total dust content.

In this review, since some other contributions in this issue have good reviews on the evolution of the total dust mass, we focus on the importance of grain size distributions and the effects of various processes on them. In particular, we put particular emphasis on the effect of various processes on extinction curves, since extinction curves give a useful clue for the grain size distribution as mentioned above. Although infrared emission (especially, in the mid-infrared) could also constrain the grain size distribution [23-25], we do not mention it in this review. The metal depletion in the ISM also provides meaningful constraints on the interstellar processing of dust [e.g., 26-28], although we do not discuss it in this review.

\section{Basic concepts of this review}

\subsection{List of the processes considered}

For convenience, we classify dust processing into two categories: (i) a process that changes both the grain size distribution and the total dust mass; and (ii) a process that only changes the grain size distribution with the total dust mass conserved. Category (i) includes dust formation in stellar ejecta, destruction by SN shocks, and grain growth by the accretion of gas-phase metals (Section 3), while Category (ii) includes shattering and coagulation (Section 4). These processes are listed in Table 1 and illustrated in Figure 1. 


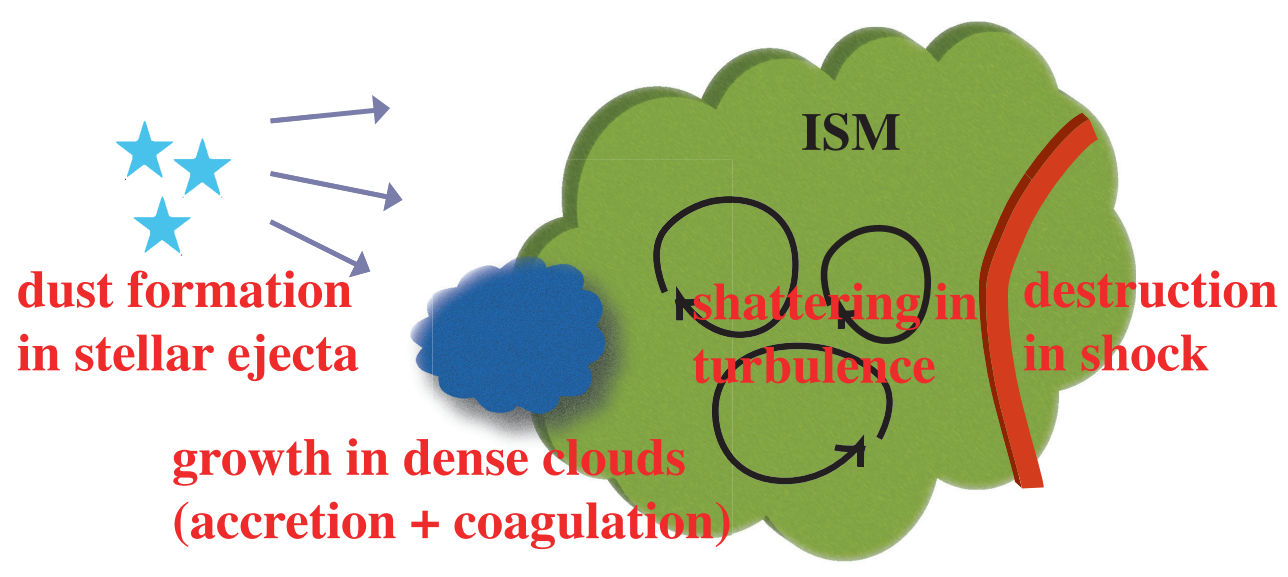

Figure 1: Main processes considered in this review. See Section 2.1 and Table 1.

Table 1: Processes considered in this review: dust formation in stellar ejecta (SNe and AGB star winds), dust destruction in interstellar SN shocks, grain growth by the accretion of gas-phase metals in the ISM, shattering (grain disruption or fragmentation by grain-grain collision), and coagulation (grain-grain sticking).

\begin{tabular}{llccc}
\hline Category $^{\mathrm{a}}$ & Process & Section $^{\mathrm{b}}$ & Dust mass $^{\mathrm{c}}$ & Extinc. $^{\mathrm{d}}$ \\
\hline \multirow{4}{*}{ (i) } & Stellar ejecta & 3.1 & $\nearrow$ & flat \\
& Shock destruction & 3.2 & $\searrow$ & flat \\
& Grain growth & 3.3 & $\nearrow$ & steep \\
\hline (ii) & Shattering & 4.1 & $\longrightarrow$ & steep \\
& Coagulation & 4.2 & $\longrightarrow$ & flat \\
\hline
\end{tabular}

a(i) Processes that change both the total dust mass and the grain size distribution; (ii) processes that only change the grain size distribution with the total dust mass conserved.

${ }^{b}$ The section in which each process is mainly explained.

${ }^{\mathrm{c}}$ The contribution to the total dust mass: $\nearrow, \searrow$, and $\longrightarrow$ indicate that the process increases, decreases, and keeps constant the total dust mass, respectively.

dEffects on the extinction curve: "flat" and "steep" indicate that the process steepens and flattens the extinction curve, respectively. See also the explanations in the text.

We neglect other possible dust formation and destruction processes in this review, since their significance is uncertain compared with the processes listed above. Dust may be destroyed by strong or hard ultraviolet (UV) radiation, but UV radiation is probably less efficient in dust destruction than SN shocks [29]. Draine \& Salpeter [29] also mention the lack of experimental knowledge on this process. UV radiation may rather destroy the local bond structures within grains, changing the chemical structure of dust [e.g., 30]. Cosmic ray bombardment may also have similar effects 
of changing chemical properties [e.g., 31]. Dust could also form in quasar (QSO) winds [32]. However, Pipino et al. [33] argue that this dust formation mode is not efficient enough to affect the dust formation in the entire region of the galaxies. In early-type galaxies which have a hot X-ray emitting halo, sputtering by the hot gas is suggested to play a dominant role in determining the dust abundance (and probably grain size distribution) [34,35]. We also neglect this process in this review since we are interested in star-forming galaxies in which the processes in the warm and cold ISM are much more important.

\subsection{Extinction curve}

One of the important points in this review is the effects of grain processing on the extinction curve. Extinction is the sum of absorption and scattering. The UV (at wavelengths longer than the Lyman limit, i.e., $\lambda>912 \AA$ ) to infrared absorption and scattering are dominated by dust grains. The extinction curve is the wavelength dependence of the extinction $\left(A_{\lambda}\right)$, which is usually quantified by the ratio (or logarithmic difference) between the intrinsic and observed brightness of an object such as a star. The slope of the extinction curve at $\lambda$ roughly reflects the abundance of grains with radii $a \sim \lambda /(2 \pi)$ [36]. Therefore, if we find a flat extinction curve at $\lambda$, namely $-\mathrm{d} \ln A_{\lambda} / \mathrm{d} \ln \lambda \ll 1$ (note that $A_{\lambda}$ is usually smaller at longer wavelengths unless there is a characteristic features such as the $2175 \AA$ bump), then the grain abundance is dominated by the grains with $a \gtrsim \lambda /(2 \pi)$. In contrast, if the extinction curve is steep, namely $-\mathrm{d} \ln A_{\lambda} / \mathrm{d} \ln \lambda$ is large, then there should be a significant abundance of small grains with $a \ll \lambda /(2 \pi)$.

\section{Processes that change both the total dust mass and the grain size distribution}

\subsection{Dust formation in stellar ejecta}

The first dust source should be the so-called Population III (Pop III) stars [37, 38]. Pop III stars are considered to be biased to massive stars, and contribute to the initial dust and metal enrichment up to $\sim 10^{-5} \mathrm{Z}_{\odot}$, at which the transition to Pop II and I stars occurs [e.g., 39, 40]. Since the subsequent major dust and metal enrichment up to the solar metallicity is driven by Pop II and I stars, we focus on these populations, that is, the stellar populations familiar to us in the nearby Universe, in this review. The most dominant dust sources are Type II supernovae (or core-collapse $\mathrm{SNe}$ ) and asymptotic giant branch (AGB) stars [e.g., 41, 42]. Below we review the size distributions of dust grains formed in those stellar ejecta.

\subsubsection{SNe II}

Type II supernovae (SNe II, hereafter simply referred to as $\mathrm{SNe}$ ), originating from massive stars with mass $>8 \mathrm{M}_{\odot}$, are considered to be the first significant grain production sources in the Universe [e.g., 37, 43] because the progenitors have short lifetimes. Some authors have attempted to calculate the dust formation by using nucleation and accretion theory of dust condensation, in which one treats the formation of stable solid nuclei out of supersaturated gas and the accretion of dust-composing elements on these nuclei [e.g., 44]. Thus, this kind of theory can predict the size distribution of each grain species formed in stellar ejecta. Dust grains condense in the adiabatic expansion phase of the SNe, in which the temperature evolution can be described as $T \propto\left(t / t_{0}\right)^{3(1-\gamma)}$, 
where $T$ is the gas temperature in the SN ejecta, $t$ is the elapsed time from the $\mathrm{SN}$ explosion, $t_{0}$ is a reference time, and $\gamma$ (generally time-variable) reflects the thermodynamical properties of the gas (see [37] for further details).

Theoretical calculations of dust condensation in SNe have been tested by high- $z$ objects [e.g., $45,46]$ as well as in nearby SNe [e.g., 47, 48]. The extinction curves in high- $z$ objects are often used as a useful probe of dust properties. Indeed, the extinction curve in a $z=6.2$ quasar, SDSS $1048+4637$ (hereafter, this extinction curve is called $z=6.2$ extinction curve), has been used to constrain the composition and size distribution of SN dust [49, 50]. Gamma-ray burst afterglows (hereafter simply called GRBs) are also useful to probe the extinction curve of their host galaxies because of their brightness and simple spectral shape [51-54]. Perley et al. [55] studied GRB 071025 and showed that the extinction curve of SN dust fits the observed spectrum. Another likely example of dust formation by $\mathrm{SNe}$ is found in Kawara et al. [56], who showed that extinction curves of dust formed in $\mathrm{SNe}$ fit the rest-frame UV-to-optical spectral energy distribution of a starburst galaxy at $z \sim 1$.

However, the dust formation models adopted above [37, 43] only consider the dust condensed in $\mathrm{SNe}$ and do not include the effect of dust destruction in the shocked region in SNe. The dust grains condensed in $\mathrm{SNe}$ should pass through the shocked region before being injected into the ISM. In the shocked region, dust grains are destroyed by sputtering [57, 58]. Nozawa et al. [58] calculated the grain trajectory by considering the gas drag and estimated the dust destruction rate by sputtering by using the relative velocity between the gas and the grain (for "nonthermal" sputtering by the supersonic motion of the grain) and the gas temperature (for "thermal" sputtering by the collision of gas particles). Then they showed that small grains are efficiently trapped in the shocked region and suffer the thermal sputtering. Thus, small grains are selectively destroyed so that the dust grains supplied from SNe are biased to large grain sizes $(a \gtrsim 0.1 \mu \mathrm{m})$. Based on their results, Hirashita et al. [59] calculated the extinction curve for a SN with a progenitor mass of $20 \mathrm{M}_{\odot}$, as shown in Figure 2a. The destruction depends on the ambient ISM density: we show the cases with ambient number densities of hydrogen nuclei $n_{\mathrm{H}}=1$ and $0.1 \mathrm{~cm}^{-3}$. We find that the extinction curve becomes flat because of the destruction of small grains. If the number density of the ISM is higher, the destruction of small grains is more efficient, so that the extinction curve is flatter. As we observe in Figure 2a, if the dust destruction in SNe is efficient, the extinction curve may be too flat to be consistent with the $z=6.2$ extinction curve, which shows a rise toward shorter wavelengths. For reference, the $z=6.2$ extinction curve is compared with the extinction curves in the Milky Way [60] and the SMC [61] in Figure 2b.

\subsubsection{AGB stars}

After a few hundreds of Myr, AGB stars start to contribute significantly to the dust enrichment in galaxies [62]. The size distribution of grains produced by AGB stars is suggested to be biased toward large $(a \gtrsim 0.1 \mu \mathrm{m})$ sizes from the observations of SEDs [63, 64], although Hofmann et al. [65] showed that the grains are not single-sized. Theoretical studies have also shown that the dust grains formed in the winds of AGB stars have typical sizes $\gtrsim 0.1 \mu \mathrm{m}$ [66-68]. There are also pieces of evidence obtained by meteoritic samples that grains such as $\mathrm{SiC}$ which are thought to originate from AGB stars from isotopic compositions $[69,70]$ have large grain sizes $(a \gtrsim 0.1 \mu \mathrm{m})$, supporting the formation of large grains in AGB stars [71, 72]. 

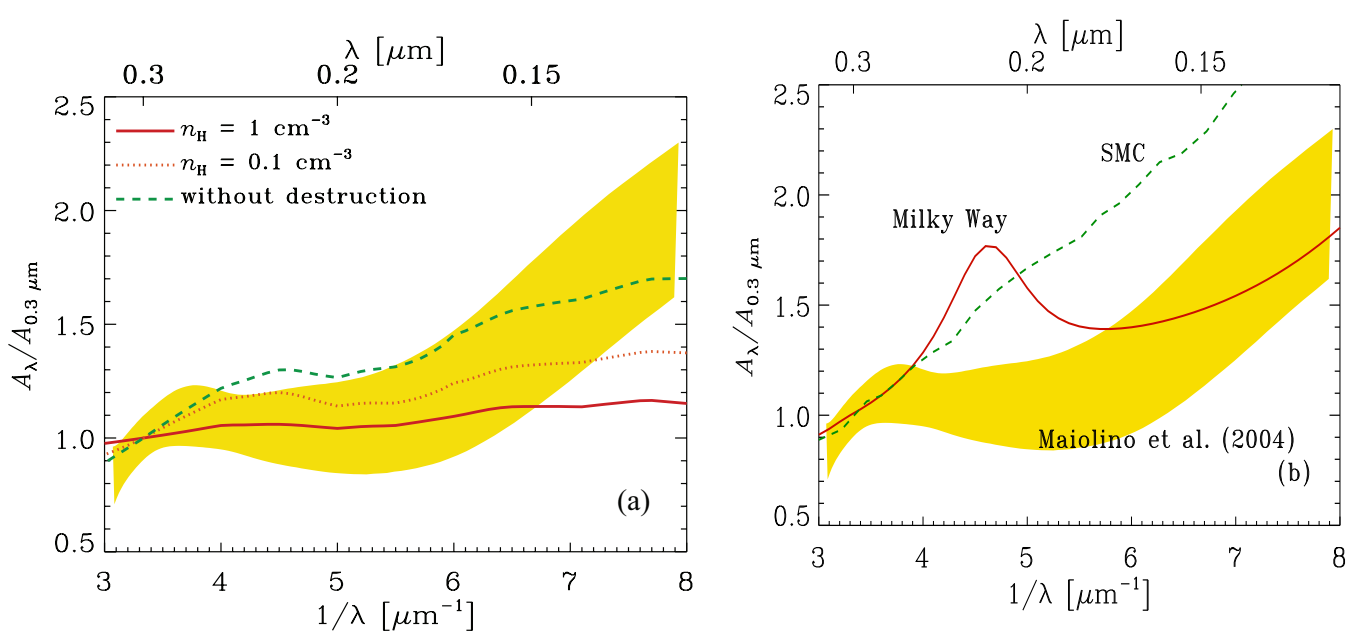

Figure 2: UV extinction curves normalized to the extinction at rest-frame wavelength $\lambda=0.3 \mu \mathrm{m}$. The range observationally derived by Maiolino et al. [49] for SDSS $1048+4637$ at $z=6.2, " z=6.2$ extinction curve", is shown by the shaded area in each panel. (a) The lines show the extinction curves of SN dust calculated by Hirashita et al. [59]. The solid and dotted lines correspond to destruction under the ambient ISM density $n_{\mathrm{H}}=1$ and $0.1 \mathrm{~cm}^{-3}$, respectively. The dashed line represents the results without destruction. (b) The $z=6.2$ extinction curve is compared with the extinction curves observed in the Milky Way [60] and the Small Magellanic Cloud [61].

\subsubsection{Flat extinction curve?}

Given that the typical grain radius of dust formed in stellar ejecta is $\gtrsim 0.1 \mu \mathrm{m}$, the optical-UV extinction curve is flat as long as stars are the only dust production source, that is, before interstellar dust processing plays a significant role [73]. In fact, the extinction curves of high-redshift $(z \sim 5)$ QSOs in Gallerani et al. [74] are flat compared with the extinction curve of the Small Magellanic Cloud (SMC), which often fit the extinction in lower-redshift QSOs [49]. Note, however, the uncertainty in deriving the extinction curves in high-redshift objects, which is arising from, for example, an assumed intrinsic spectrum [75]. As mentioned in Section 3.1.1, extinction curves predicted for the dust formed in stellar ejecta may be too flat, since the observed extinction curves in Gallerani et al. [74] has a significant rise toward shorter UV wavelengths. This discrepancy may be resolved if we consider interstellar processing (Section 4).

It is still worth noting that some high- $z$ objects have flat extinction curves. Flatness of extinction curves may explain some unreddened BAL quasars at $z>5$ (e.g., SDSS 1044+01 and SDSS 0756+41) in Maiolino et al. [76]. From submillimeter observations, they are known to have a large amount of dust $\left(\sim 10^{8} \mathrm{M}_{\odot}\right)$. Thus, the absence of reddening may be due to a flat extinction curve.

We should note that, as shown later, interstellar processing of dust is important in metalenriched systems such as QSOs even at high redshift. Thus, for the purpose of testing dust formation in stellar ejecta, it is ideal to derive extinction curves in metal-poor high-redshift galaxies, although detailed observations of such a population of galaxies are difficult. 


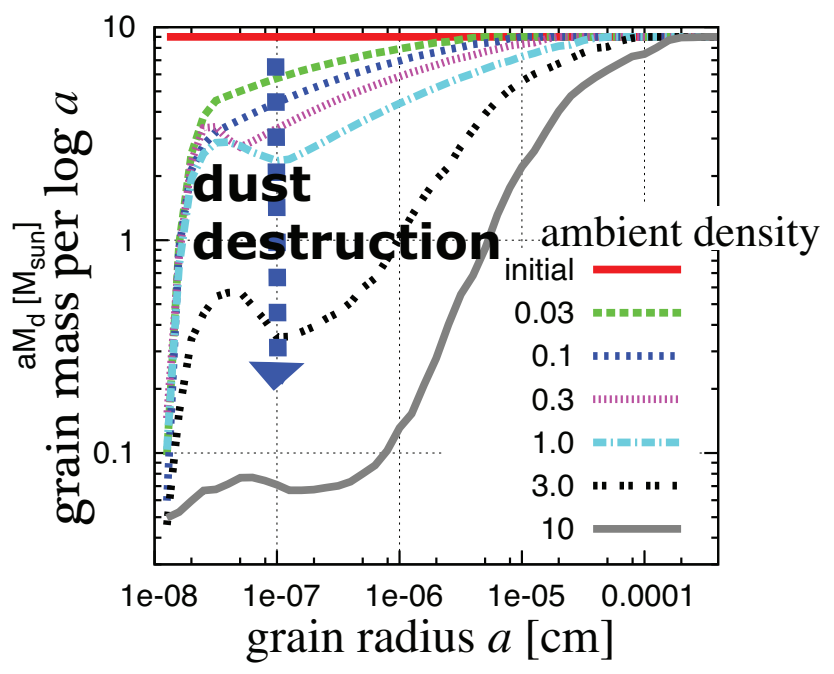

Figure 3: Change of the dust mass per logarithmic grain radius after a passage of a SN shock in the ISM, whose hydrogen number densities are $0.03-10 \mathrm{~cm}^{-3}$ as indicated in the figure [22]. To show the dependence on the grain radius, we assume a flat (constant) grain size distribution. We observe that the effect of shock destruction is the most significant at small grain sizes and in high ambient densities.

\subsection{Dust destruction by interstellar shocks}

Dust grains, after being injected into the ISM, are destroyed by sputtering if they are swept by SN shocks. We can neglect the thermal evaporation of dust because the temperature of dust hardly rises up to its sublimation temperature [77]. Once dust grains enter a SN shock, the relative velocity between gas and dust emerges, since the dust grains move ballistically while the gas is decelerated. As a result, the dust grains suffer nonthermal sputtering. Small grains $(a \lesssim 0.01 \mu \mathrm{m})$ are quickly decelerated by gas drag and trapped in the hot gas, being destroyed by thermal sputtering [78]. Because thermal sputtering is a surface process, the dust destruction timescale by thermal sputtering is proportional to the grain radius [34]. Moreover, large grains stay in the inner region of the postshock flow where the gas density is lower than that near the shock front, so that they are less destroyed than small grains [78]. For the reasons above, small grains are lost more than large grains after the passage of SN shocks as shown in Figure 3 [see also 22]. Therefore, the extinction curve after the destruction by SN shocks becomes flatter [e.g., 79].

There are some pieces of evidence that dust grains are indeed processed by SN shocks. Andersen et al. [80] analyzed Spitzer mid-infrared data of a sample of Galactic SN remnants (SNRs). They found low abundance of small grains for two SNRs, which is likely to be due to sputtering. On the other hand, they also found enhancement of the abundance of very small grains relative to large grains for SNRs interacting with molecular clouds. This is probably due to shattering. Seok et al. [81] detected polycyclic aromatic hydrocarbons (PAH) in SNRs interacting with molecular clouds, which could be interpreted as PAH formation by fragmentation or shattering of large grains. Jones et al. [14], by using their theoretical models, show that sputtering and shattering both occur in SN shocks [see also 82]. Further observational evidence of grain processing in SNRs will be an interesting topic in the future. 
We estimate the timescale of dust destruction by the sputtering in SN shocks in a galaxy, $\tau_{\mathrm{SN}}$, following McKee [83] as $\tau_{\mathrm{SN}}=M_{\mathrm{gas}} /\left(\varepsilon_{\mathrm{s}} M_{\mathrm{s}} \gamma\right)$, where $\varepsilon_{\mathrm{s}}$ and $M_{\mathrm{s}}$ are the dust destruction efficiency and the gas mass swept by a single high-velocity $\left(>100 \mathrm{~km} \mathrm{~s}^{-1}\right) \mathrm{SN}$ blast, respectively, and $\gamma$ is the $\mathrm{SN}$ rate. Since the lifetime of SN progenitor is usually much shorter than the galaxy evolution timescale, it is assumed that the $\mathrm{SN}$ rate is proportional to the star formation rate. The efficiency $\varepsilon_{\mathrm{S}}$ is around 0.1 and the mass swept by a single blast is estimated as $M_{\mathrm{S}} \sim E_{\mathrm{SN}} / v_{\mathrm{S}}^{2}$, where $E_{\mathrm{SN}} \sim 10^{51}$ erg is the energy injected from the supernova and $v_{\mathrm{S}} \sim 100 \mathrm{~km} \mathrm{~s}^{-1}$ is the velocity of the blast. We adopt $\varepsilon_{\mathrm{S}} M_{\mathrm{S}}=1300 \mathrm{M}_{\odot}$ [83]. Then we obtain $\tau_{\mathrm{SN}}=M_{\mathrm{gas}} /\left(\beta_{\mathrm{SN}} \psi\right)$, where $\psi$ is the star formation rate, and $\beta_{\mathrm{SN}} \equiv \varepsilon_{\mathrm{S}} M_{\mathrm{s}} \gamma / \psi=9.65$. As pointed out by Jones $\&$ Nuth [84], $\varepsilon_{\mathrm{s}}$ is uncertain by a factor of $\sim 2$ and is dependent on the assumed grain composition [see also 85]. In the Milky Way, $M_{\text {gas }} \sim 5 \times 10^{9} \mathrm{M}_{\odot}[86,87]$ and $\psi \sim 1.3 \mathrm{M}_{\odot} \mathrm{yr}^{-1}$ [88], so we obtain $\tau_{\mathrm{SN}} \sim 4 \times 10^{8} \mathrm{yr}$ for the Milky Way.

As noted by many authors, this timescale is significantly shorter than the timescale of dust supply from stars [e.g., 83]. The dust supply from stars occurs on a timescale of metal enrichment, which is roughly estimated by the gas consumption timescale ( $\sim$ the timescale of ISM recycling): $\tau_{\mathrm{SF}} \sim M_{\mathrm{gas}} / \psi \sim 3 \times 10^{9} \mathrm{yr}$ in the Milky Way. This is much longer than the dust destruction timescale. Moreover, the ratio between $\tau_{\mathrm{SN}}$ and $\tau_{\mathrm{SF}}$ is always $\beta_{\mathrm{SN}} \sim 10$, which is insensitive to the star formation activity. Therefore, we expect that the dust enrichment timescale up to the Galactic dust-to-gas ratio is generally much slower than the dust destruction by SN shocks. This is why we need to consider a dust formation mechanism other than the condensation in stellar ejecta.

\subsection{Grain growth by accretion}

The most promising candidate of a dust formation process other than the condensation in stellar ejecta is grain growth by the accretion of gas-phase metals. This process is simply referred to as accretion in this review. Various models that deal with dust enrichment in galaxies include accretion $[6,8-10,33,89,90]$. Accretion is motivated not only by the quick dust destruction as explained in Section 3.2, but also by the observations of huge amounts of dust $\left(\sim 10^{8} \mathrm{M}_{\odot}\right)$ in high$z$ quasars [91-95]. Some theoretical studies have shown difficulty in reproducing such large dust content only by the stellar origin of dust $[89,96,97]$.

Accretion occurs most efficiently in dense regions in the ISM such as the cold neutral medium and molecular clouds. Grain growth by accretion occurs efficiently only after the ISM is significantly enriched with dust and metals, since the grain growth rate is proportional to the metallicity. Inoue [98] and Asano et al. [90] showed that accretion becomes the dominant mechanism of grain formation over dust condensation in stellar ejecta at a certain metallicity referred to as the critical metallicity for grain growth, which is typically a sub-solar metallicity.

It has been shown that accretion dramatically changes the grain size distribution and extinction curve $[99,100]$. Hirashita [99] studied the effect of accretion and coagulation (for coagulation see Section 4.2), since both processes occur simultaneously in the dense ISM. In Figure 4, we show the time evolution of grain size distribution by both accretion and coagulation, only accretion and only coagulation. We adopt a power-law grain size distribution $\left(n(a) \propto a^{-3.5}\right)$, which reproduces the Milky Way extinction curve, for the initial condition. Since accretion is a surface process, the impact of accretion is significant at small sizes, where the surface-to-volume ratio is large. After all 

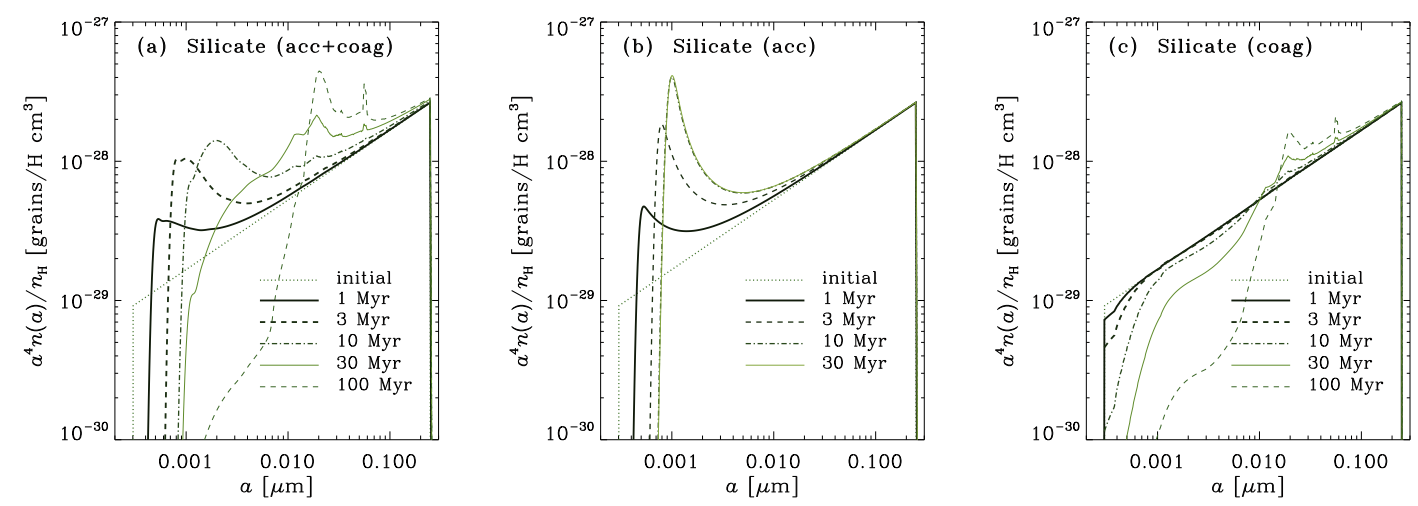

Figure 4: Time evolution of grain size distribution calculated by Hirashita [99] with a power-law initial condition $n(a) \propto a^{-3.5}$ (see the text). The thick solid, thick dashed, dot-dashed, thin solid and thin dashed lines show the grain size distributions at $t=1,3,10,30$, and $100 \mathrm{Myr}$, respectively (in panel $\mathrm{b}$, since the grain size distributions are the same for $t \geq 10 \mathrm{Myr}$, we do not show the size distribution at $t=100 \mathrm{Myr}$ ). Each panel shows the case with (a) both accretion and coagulation, (b) only accretion and (c) only coagulation.

the gas-phase metals are used up, accretion stops (see panel b in Figure 4). Carbonaceous dust also behaves similarly in accretion as long as we start from the same power-law grain size distribution.

The effects of accretion and coagulation on the extinction curve are shown in Figure 5 for the grain size distributions at $t=10 \mathrm{Myr}$. The extinction curves are calculated by using the optical constants for astronomical silicate and graphite in Draine \& Lee [101]. We observe that accretion increases the grain opacity at all wavelengths because the grain mass grows. In order to examine the wavelength dependence of how the grain size distribution is affected by accretion and coagulation, we also show the ratio between the extinction curve at $t=10 \mathrm{Myr}$ to that at $t=0 \mathrm{Myr}$ (initial) in the lower panel. We find that accretion steepens the extinction curve. This is contrary to a naive expectation that large grains show flat extinction curves. This "contradiction" is explained as follows. The extinction at a wavelength $\lambda$ is proportional to $a^{3}$ if $a \ll \lambda$, while it is proportional to $a^{2}$ (i.e., less sensitive to the grain size) if $a \gtrsim \lambda /(2 \pi)$ [36]. In other words, the UV extinction is more sensitive to the enhancement of small grains than the extinction at longer wavelengths. Therefore, the extinction at shorter wavelengths increases more sensitively as a result of accretion. The $0.22-\mu \mathrm{m}$ bump, which is due to small graphite grains in the model, also becomes more prominent after accretion. At optical and near-infrared wavelengths, the extinction is relatively insensitive compared to other wavelengths, since it is more affected by the largest grains intact after accretion and coagulation. As $\lambda$ becomes large at mid-infrared and longer wavelengths (i.e. $\lambda \gg 2 \pi a_{\max }$ ), the extinction is just proportional to the grain mass (or volume).

The steepening of extinction curve by accretion occurs if there are abundant small grains with $a \lesssim \lambda /(2 \pi)$. If all grains are larger than $\sim \lambda /(2 \pi)$, accretion flattens the extinction curve rather than steepens it [79]. However, if all grains are larger than $\sim \lambda /(2 \pi)$, the extinction curve is flat anyway. Therefore, flattening of extinction curve by accretion does not have importance in shaping the extinction curve in such a case. 


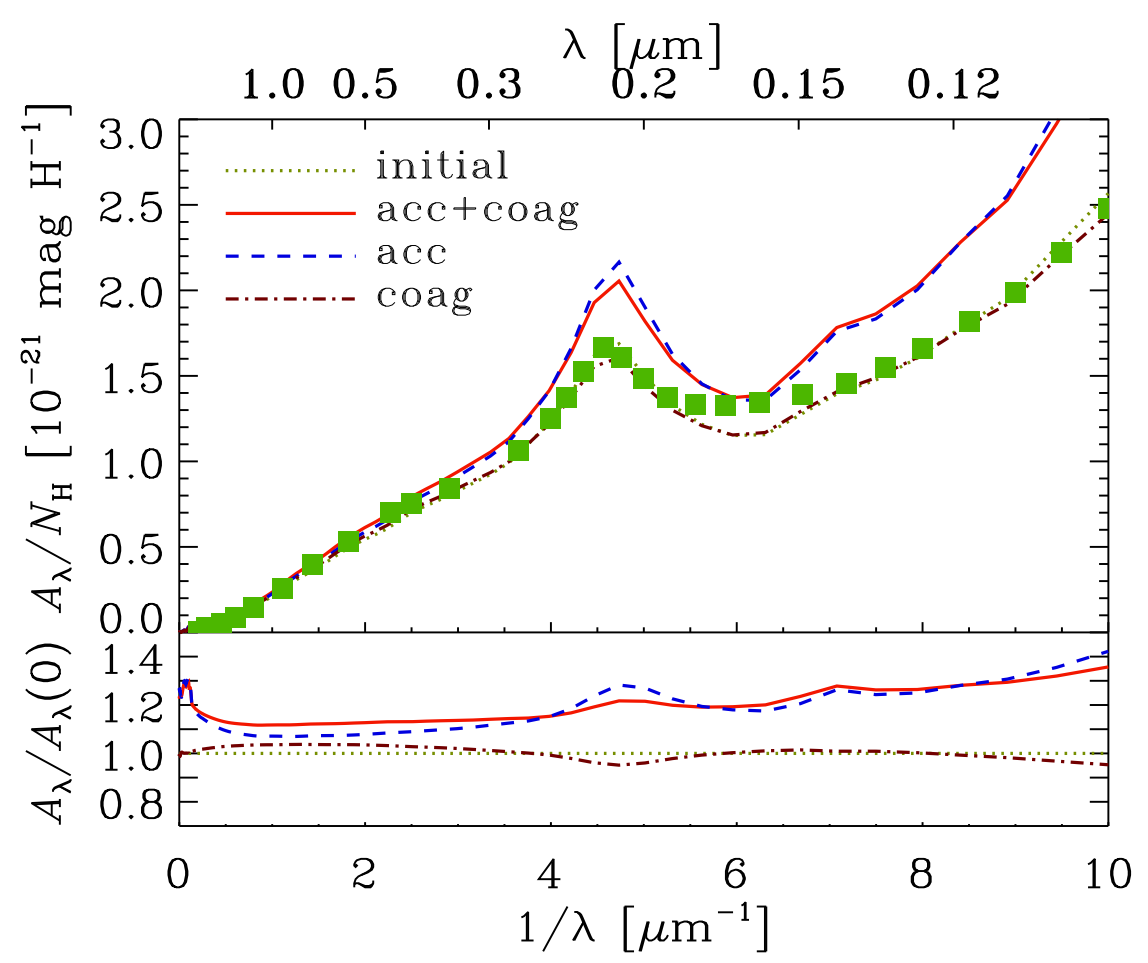

Figure 5: Upper panel: extinction curves in units of magnitude per hydrogen calculated by Hirashita [99]. The dotted line (almost identical to the dot-dashed line) is the initial extinction curve before accretion and coagulation. The solid, dashed and dot-dashed lines show the results with both accretion and coagulation, only accretion and only coagulation, respectively, at $t=10 \mathrm{Myr}$. The filled squares represent the Milky Way extinction data taken from Pei [61]. Lower panel: the extinction divided by the initial extinction. The correspondence between the line types and the models is the same as above.

\section{Processes that only changes the grain size distribution but does not change the total dust mass}

\subsection{Shattering}

Stellar dust production and dust destruction in SN shocks both predict flat extinction curves, and grain growth steepens the extinction curves only if there are abundant small grains. This means that, if we only consider the processes that directly change the total dust mass (i.e., the processes considered in Section 3) significantly steep extinction curves such as the ones observed in the Milky Way and the Magellanic Clouds cannot be realized. Moreover, as shown in Figure 2, the $z=6.2$ extinction curve is not completely flat especially in the far-UV.

As mentioned in Section 3.2, both sputtering and shattering occur in SN shocks [14]. The importance of shattering is more pronounced, considering that interstellar turbulence can activate grain motion through gas drag and gyroresonance (resonance between the gyro-motion of charged grains around magnetic fields and the gas motion associated with magnetohydrodynamical waves) [102]. In particular, larger grains tend to acquire larger velocities, since they tend to couple with larger-scale gas motions which have larger velocities [102]. In the diffuse ISM, grains with $a \gtrsim$ 

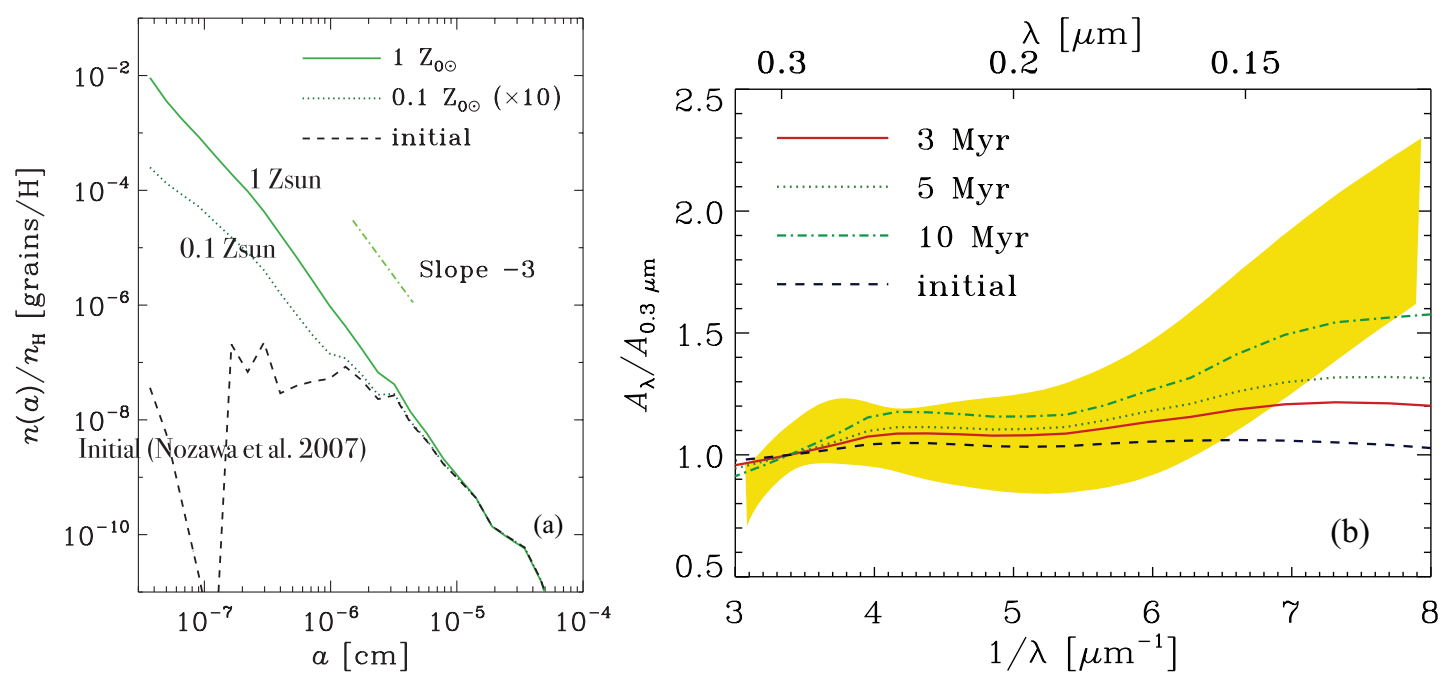

Figure 6: Left: Variation of grain size distribution by shattering in a $\operatorname{WIM}\left(n_{\mathrm{H}}=1 \mathrm{~cm}^{-3}\right.$ and $\left.T=8000 \mathrm{~K}\right)$. A shattering duration of $5 \mathrm{Myr}$ is assumed, and two cases for the oxygen abundance ( 1 and 0.1 solar; solid and dotted lines, respectively) are examined (note that the oxygen abundance is proportional to the dust abundance). The dashed line shows the initial condition (dust injected into the ISM from a SNe II; Nozawa et al. 58). Right: Evolution of extinction curve by shattering in a WIM $\left(n_{\mathrm{H}}=1 \mathrm{~cm}^{-3}, T=8000 \mathrm{~K}\right.$, and solar oxygen abundance). The extinction curve is normalized to the value at $0.3 \mu \mathrm{m}\left(A_{0.3} \mu \mathrm{m}\right)$. The shaded region shows the $z=6.2$ extinction curve [49]. The solid, dotted, and dot-dashed lines present the extinction curves for various shattering durations ( 3,5 , and $10 \mathrm{Myr}$, respectively). The initial extinction curve is shown by the dashed line.

$0.1 \mu \mathrm{m}$ can acquire velocity dispersions as large as $\sim 10 \mathrm{~km} \mathrm{~s}^{-1}$. Under such large velocity dispersions, the grains are shattered in grain-grain collisions [103]. Hirashita et al. [104] examined the effect of shattering in the warm ionized medium on the dust injected from $\mathrm{SNe}$ ( $\mathrm{SN}$ dust). They adopted the grain size distribution of dust ejected from a SN whose progenitor has a mass of $20 \mathrm{M}_{\odot}$ (shock destruction in the SN is considered under an ambient hydrogen number density of $n_{\mathrm{H}}=1 \mathrm{~cm}^{-3}$; Nozawa et al. 58) as an initial condition. The result is shown in Figure 6. We find that, although the grains ejected from SNe are expected to be biased to large sizes $(a \gtrsim 0.1 \mu \mathrm{m})$ because of the shock destruction in the $\mathrm{SNe}$, the shattering in the warm ionized medium is efficient enough to produce small grains in several Myr. The shattering efficiency depends on the grain abundance, which is assumed to be proportional to metallicity (compare the solid and dotted lines in Figure 6a). In Figure 6b, we show the extinction curve for various shattering durations. We observe that the production of small grains by shattering contributes to the steepening of the extinction curve. Thus, we conclude that, if the metallicity is nearly or above solar, shattering is efficient enough to modify the extinction curve in several Myr.

Shattering is a unique mechanism of small grain formation. The property of shattering predicts a top-down evolutionary nature of grain sizes. Seok et al. [105] applied this top-down concept for the calculation of the evolution of PAHs in a galaxy by assuming that PAHs form as a result of shattering of large carbonaceous grains [106]. They succeeded in reproducing the observed strong metallicity dependence of PAH abundance. This metallicity dependence comes from the dependence of the total carbonaceous dust abundance on the metallicity. Note that the main PAH 
formation mechanism is still a matter of debate; for example, Galliano et al. [107] explained the metallicity dependence of PAH abundance by the delayed injection of PAHs from AGB stars. Gordon et al. [108] pointed out the importance of processing by radiation based on the correlation between ionization indicator and PAH emission strength.

\subsection{Coagulation}

As shown in Figure 4, coagulation pushes the grain size distribution toward larger grain sizes. In particular, coagulation is a unique mechanism that can create large grains, since accretion predominantly works at small grain sizes. As a result of coagulation, the extinction curve becomes flatter (Fig. 5; see also [100]). Note that accretion rather steepens the extinction curve; thus, coagulation has a different influence on the extinction curve compared to accretion.

The existence of large micron-sized grains is observed as mid-infrared glow of dense molecular cloud cores, the so-called "coreshine," which is thought to come from scattering by micronsized grains $[109,110]$. As mentioned above, coagulation is of unique importance in creating large grains. Based on numerical calculations of coagulation starting from the typical grain size distribution in the diffuse ISM, Ormel et al. [111] and Hirashita \& Li [112] showed that the time required for the grains to grow up to $\sim 1 \mu \mathrm{m}$ is a few times the free-fall timescale. This indicates that those dense cores observed in coreshine are relatively long-lived entities in molecular clouds, rather than dynamically transient objects that last for one free-fall time or less.

\section{Dust enrichment history of a galaxy}

The evolution of the total dust content in a galaxy can be modeled by using a "chemical evolution" framework, which calculates the metal enrichment in a consistent manner with the recycling of the ISM by star formation and stellar death [113]. The evolution of dust content can also be added successfully by including the processes described in Section 3. Important features of the dust enrichment can be caught successfully if we present the relation between dust-to-gas ratio and metallicity $[7,8,12,114,115]$. We show the relation calculated by Hirashita \& Kuo [116] as an example in Figure 7. The general features predicted by such a model is that dust is predominantly supplied by stellar ejecta at low metallicity while it increases by the accretion of gas-phase metals after the system reaches a certain metallicity ("critical metallicity" in Section 3.3), which depends on the grain size distribution. Because of dust destruction by SN shocks, accretion is crucial to explain the dust-to-gas ratios in nearby galaxies (Section 3.2). For the modeling and general features of model calculations, see e.g., Zhukovska et al. [10] and Asano et al. [90].

Although the processes listed in Section 3 directly drive the evolution of the total dust content, the processes explained in Section 4 also affect the total dust content indirectly, since the grain size distribution affects dust formation and destruction processes. In particular, as shown by Kuo \& Hirashita [97], dust growth by accretion is significantly affected by the grain size distribution. Figure 8 shows their result. They examined two cases for the grain size distribution: one is the grain size distribution of dust produced by $\mathrm{SNe}$ (SN dust), and the other is the one after shattering (both grain size distributions are shown in Figure 6). Since the SN dust is biased toward large grains whose surface-to-volume ratio is small, the surface process such as accretion is inefficient; as a result, the increase of the dust-to-gas ratio occurs only at super-solar metallicities, failing to 


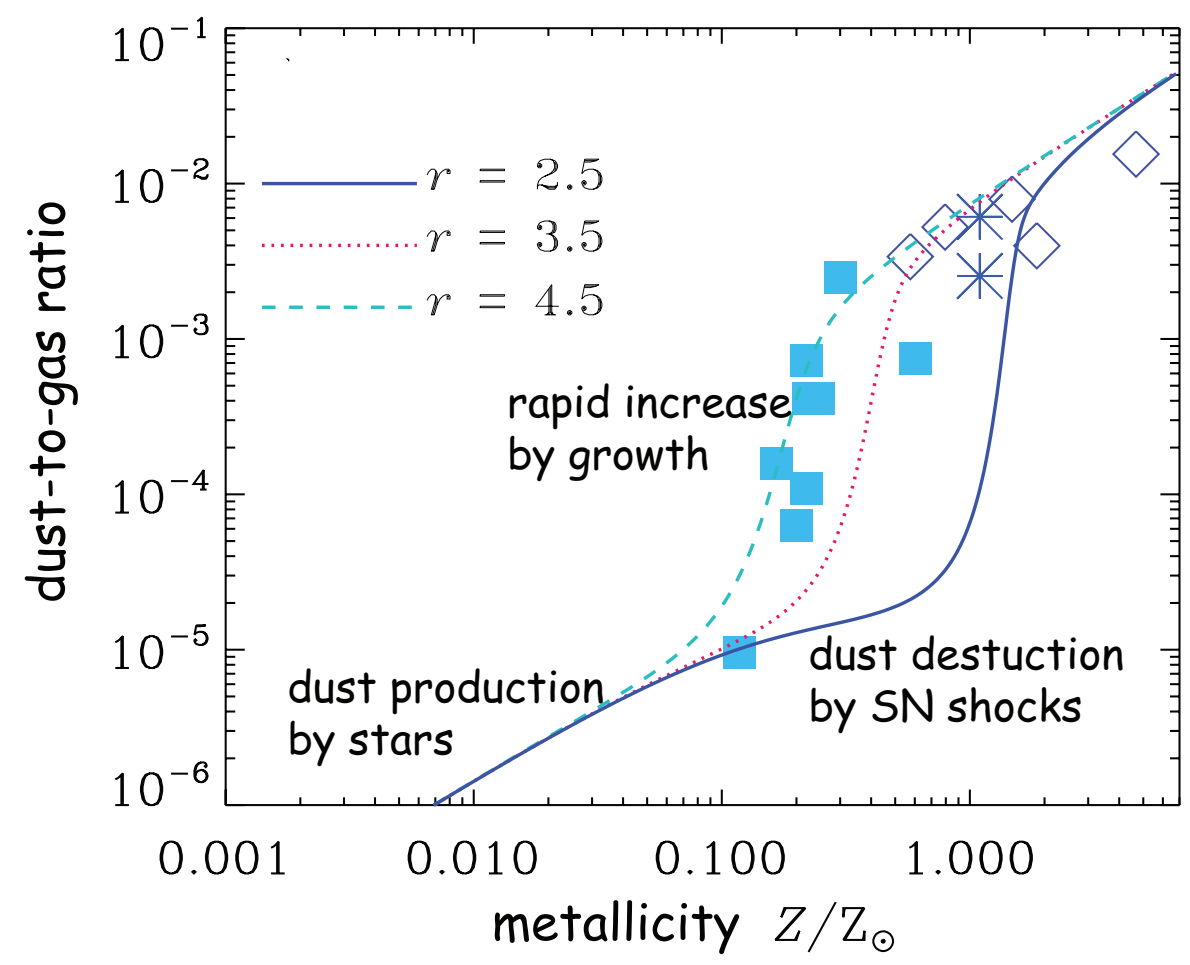

Figure 7: Relation between dust-to-gas ratio and metallicity calculated by Hirashita \& Kuo [116]. At low metallicity, both dust and metals are produced by stars, and the relation is linear. The dust destruction by SN shocks decelerates the increase of dust-to-gas ratio. At a certain metallicity, grain growth by the accretion of gas-phase metals rapidly raise the dust-to-gas ratio, which saturates when a large part of gas-phase metals are locked into dust. Hirashita \& Kuo [116] considered power-law grains size distributions with a power index of $-r: r=4.5$ (2.5) represents the case in which small grains are the most (least) abundant. The grain growth begins early if small grains are dominated. This is because of the large surface-to-volume ratio of small grains. We also show various observational data for comparison [see 116, for the references].

explain the observed dust-to-gas ratio in high- $z$ QSOs. On the other hand, if we take the increase of small grains by shattering into account, the observed dust-to-gas ratio in high- $z$ QSOs can be naturally explained because accretion increases the dust-to-gas ratio at lower metallicity. Therefore, we conclude that the grain size distribution has a large impact on dust enrichment in galaxies.

Recently, Asano et al. [79] has incorporated all the processes in this review. They pointed out some interesting interplay between the different processes. They first confirmed that the small grain production by shattering activates the grain growth by accretion. They also found that the grain growth by accretion creates a bump in the grain size distribution around $a \sim 0.001-0.01$ $\mu \mathrm{m}$. Because of this bump, the extinction curve becomes too steep to match the ones observed in nearby galaxies. This bump is only eliminated by coagulation, so that they also assumed strong coagulation. Such strong coagulation efficiently creates large $(a \gtrsim 0.1 \mu \mathrm{m})$ grains, which are eventually shattered and redistributed at small grain sizes. As a result, this strong interplay between shattering and coagulation predicts a power-law like grain size distribution (see also [117]), nearly reproducing the Milky Way extinction curve. 


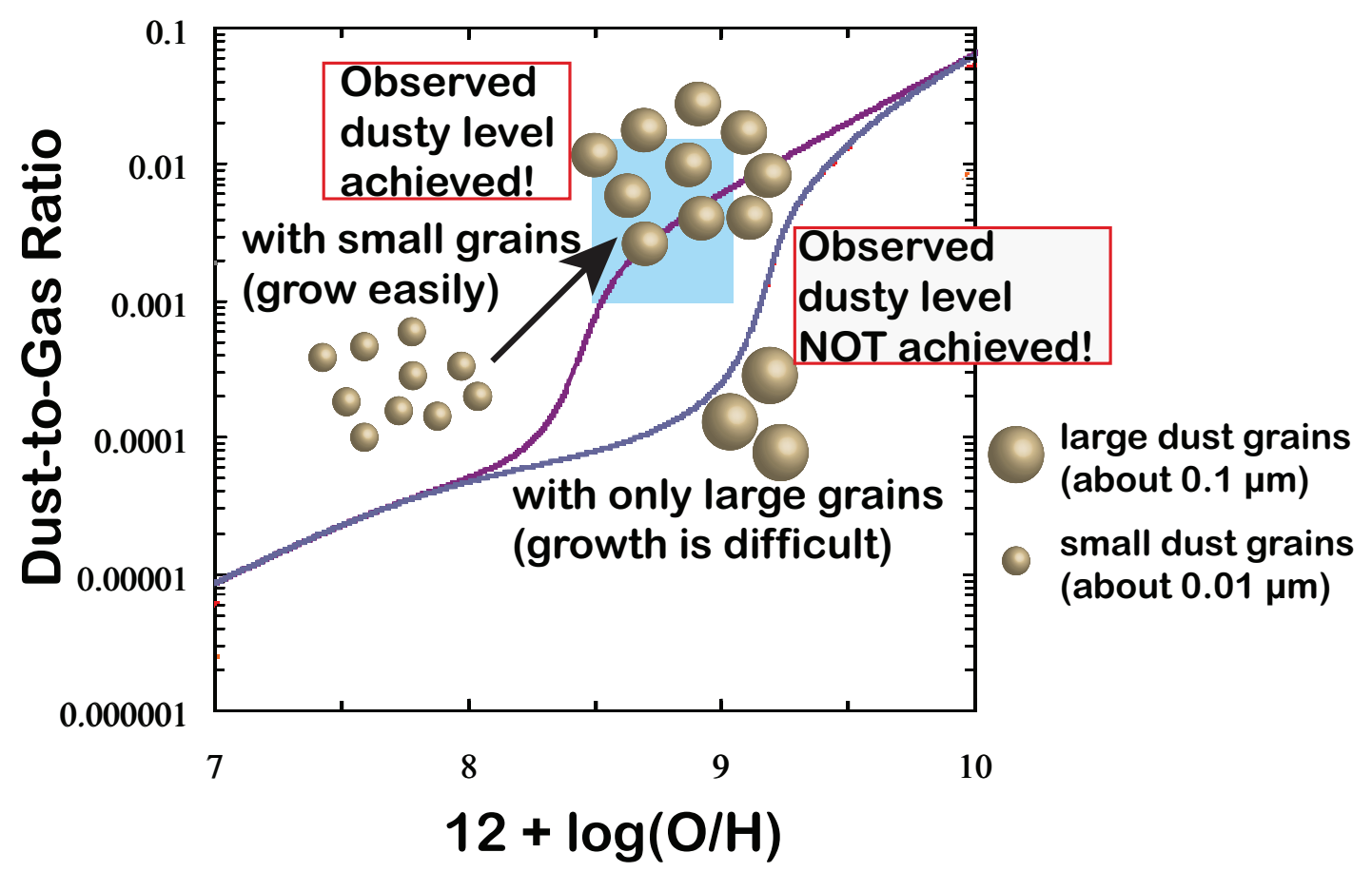

Figure 8: Dust-to-gas ratio as a function of metallicity calculated by Kuo \& Hirashita [97]. The metallicity is indicated by the oxygen abundance, $12+\log (\mathrm{O} / \mathrm{H})$. The upper and lower lines show the evolutionary tracks for two grain size distributions: one is for the dust ejected from SNe before shattering (dashed line in Figure 6a) and the other is after shattering (solid line in Figure 6a). The shaded region is the observationally reasonable region for high-redshift QSOs at $z \gtrsim 5$ [94]. We put illustration of dust growth along the model predictions. The dust ejected from $\mathrm{SNe}$ is biased toward large grain sizes, so accretion is inefficient. As a result, the observed large dust-to-gas ratios observed in those high-redshift QSOs are difficult to achieve. The increase of small grains by shattering nicely reproduces the observed high dust-to-gas ratio because of efficient accretion.

\section{Conclusion}

We have reviewed important processes for the evolution of dust abundance and grain size distribution in galaxies. The processes are dust formation in stellar ejecta, mainly supernovae (SNe) and asymptotic giant branch star winds, dust destruction in supernova (SN) shocks in the interstellar medium (ISM), dust growth by the accretion of gas-phase metals in the dense ISM, shattering in the diffuse ISM and coagulation in the dense ISM. The dust formation in stellar ejecta is the most important process for the first dust enrichment in galaxies, the dust enrichment being somewhat decelerated by the shock destruction. Since dust supplied from stellar ejecta is biased toward large sizes, the extinction curve is predicted to be flat. The extinction curve becomes steep only if we consider shattering. Moreover, after a large abundance of small grains are produced by shattering, grain growth by accretion further steepens the extinction curve. The effects of each process on the grain size distribution is shown in Figure 9, and the effects on the total dust mass (abundance) and extinction curve are listed in Table 1.

The interstellar origin of the dust, i.e., the accretion of gas-phase metals on to preexisting 


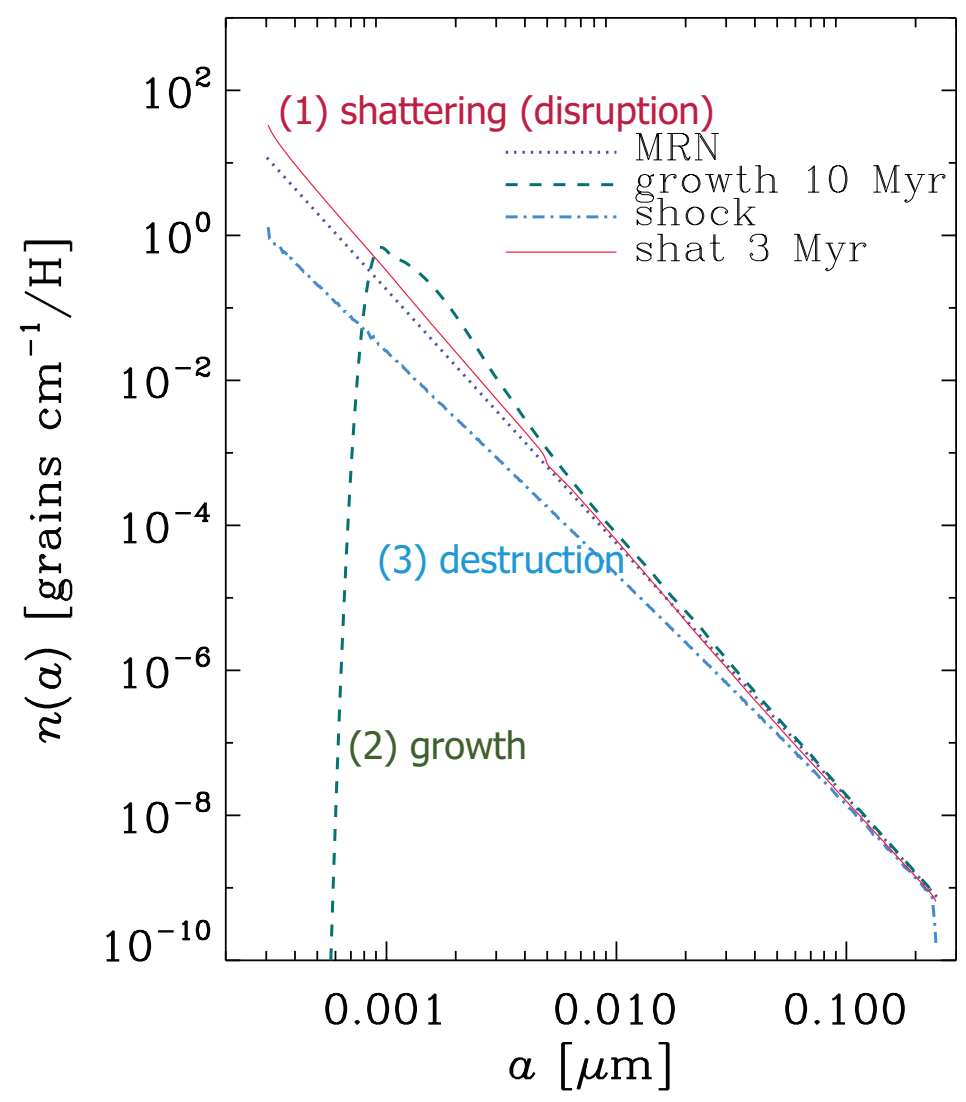

Figure 9: Effects of various processes on the grain size distribution (per hydrogen nucleus) as a summary. See Hirashita \& Nozawa [118] for details of calculations. The initial condition (dotted line) is a power-law with a power index of -3.5 [19] and an upper and lower grain radius of 0.003 and $0.25 \mu \mathrm{m}$, respectively, which broadly reproduces the extinction curves in the Milky Way and the Magellanic Clouds [61]. We show the results for silicate. The solid line shows the size distribution after shattering in the warm ionized medium for 3 Myr. Shattering enhances the abundance of small grains with a slight decrease in the large-grain abundance. The total dust mass is conserved in shattering. The dashed line shows grain growth by accretion and coagulation in dense medium with a duration of $10 \mathrm{Myr}$. Accretion is significant at the smallest sizes while coagulation pushes the grains to large sizes. The total dust mass increases by accretion. The dotdashed line shows destruction by supernova shocks. The effect is significant at small grain sizes and the total dust mass decreases by this process.

dust, is the dominant driver of dust enrichment above a certain metallicity referred to as the critical metallicity of dust growth. This critical metallicity depends on the grain size distribution. Therefore, the change of the grain size distribution by interstellar processing affects the dust enrichment. In particular, it has been shown that the small grain production by shattering significantly activates the grain growth, naturally explaining the high dust abundance in high- $z$ QSOs. This is an example of interplay between two processes. Coagulation and shattering may also interplay to produce a power-law-like grain size distribution, which could successfully explain the extinction curves in nearby galaxies. 


\section{Acknowledgments}

The author thanks the support from the Ministry of Science and Technology (MOST) grant 102-2119-M-001-006-MY3.

\section{References}

[1] Désert, F.-X., Boulanger, F., \& Puget, J. L. 1990, A\&A, 237, 215

[2] Silva, L., Granato, G. L., Bressan, A., \& Danese, L. 1998, ApJ, 509, 103

[3] Yajima, H., Umemura, M., \& Mori, M. 2012, MNRAS, 420, 3381

[4] Gould, R. J., \& Salpeter, E. E. 1963, ApJ, 138, 393

[5] Cazaux, S., \& Tielens, A. G. G. M. 2002, ApJ, 575, L29

[6] Dwek, E. 1998, ApJ, 501, 643

[7] Lisenfeld, U., \& Ferrara, A. 1998, ApJ ,496, 145

[8] Hirashita, H. 1999, ApJ, 510, L99

[9] Inoue, A. K. 2003, PASJ, 55, 901

[10] Zhukovska, S., Gali, H.-P., \& Trieloff, M. 2008, A\&A, 479, 453

[11] Mattsson, L., \& Andersen, A. C. 2012, MNRAS, 423, 38

[12] Kuo, T.-M., Hirashita, H., \& Zafar, T. 2013, MNRAS, 436, 1238

[13] Borkowski, K. J., \& Dwek, E. 1995, ApJ, 454, 254

[14] Jones, A. P., Tielens, A. G. G. M., \& Hollenbach, D. J. 1996, ApJ, 469, 740

[15] Ossenkopf, V. 1993, A\&A, 280, 617

[16] Chokshi, A., Tielens, A. G. G. M., \& Hollenbach, D. 1993, ApJ, 407, 806

[17] Dominik, C., \& Tielens, A. G. G. M. 1997, ApJ, 480, 647

[18] Hoyle, F., \& Wickramasinghe, N. C. 1969, Nature, 223, 450

[19] Mathis, J. S., Rumpl, W., \& Nordsieck, K. H. 1977, ApJ, 217, 425

[20] Kim, S.-H., Martin, P. G., \& Hendry, P. D. 1994, ApJ, 422, 164

[21] Weingartner, J. C., \& Draine, B. T. 2001, ApJ, 548, 296

[22] Yamasawa, D., Habe, A., Kozasa, T., Nozawa, T., Hirashita, H., Umeda, H., \& Nomoto, K. 2011, ApJ, 735, 44 
[23] Draine, B. T., \& Anderson, N. 1985, ApJ, 292, 494

[24] Li, A., \& Draine, B. T. 2001, ApJ, 554, 778

[25] Takeuchi, T. T., Ishii, T. T., Nozawa, T., Kozasa, T., Hirashita, H. 2005, MNRAS, 362, 592

[26] O’Donnell, J. E., \& Mathis, J. S. 1997, ApJ, 479, 806

[27] Weingartner, J. C., \& Draine, B. T. 1999, ApJ, 517, 292

[28] Voshchinnikov, N. V., \& Henning, Th. 2010, A\&A, 517, A45

[29] Draine, B. T., \& Salpeter, E. E. 1979a, ApJ, 231, 438

[30] Gadallah, K. A. K., Mutschke, H., \& Jäger, C. 2012, A\&A, 544, A107

[31] Mennella, V., Baratta, G. A., Esposito, A., Ferini, G., \& Pendleton, Y. J. 2003, ApJ, 587, 727

[32] Elvis, M., Marengo, M., \& Karovska, M. 2002, ApJ, 567, L107

[33] Pipino, A., et al. 2011, A\&A, 525, A61

[34] Draine, B. T., \& Salpeter, E. E. 1979b, ApJ, 231, 77

[35] Patil, M. K., Pandey, S. K., Sahu, D. K., \& Kembhavi, A. 2007, A\&A, 461, 103

[36] Bohren, C. F., \& Huffman, D. R. 1983, Absorption and Scattering of Light by Small Particles. Wiley, New York

[37] Nozawa, T., Kozasa, T., Umeda, H., Maeda, K., Nomoto, K. 2003, ApJ, 598, 785

[38] Schneider, R., Ferrara, A., \& Salvaterra, R. 2004, MNRAS, 351, 1379

[39] Omukai, K., Tsuribe, T., Schneider, R., \& Ferrara, A. 2005, ApJ, 626, 627

[40] Schneider, R., Omukai, K., Inoue, A. K., \& Ferrara, A. 2006, MNRAS, 369, 1437

[41] Gehrz, R. 1989, in Interstellar Dust, Proc. IAU Symp. 135, L. J. Allamandola \& A. G. G. M. Tielens eds., Kluwer, Dordrecht, p. 445

[42] Matsuura, M., et al. 2009, MNRAS, 396, 918

[43] Todini, P., \& Ferrara, A. 2001, MNRAS, 325, 726

[44] Kozasa, T., Hasegawa, H., \& Nomoto, K. 1989, ApJ, 344, 325

[45] Gall, C., Andersen, A. C., \& Hjorth, J. 2011a, A\&A, 528, A14

[46] Gall, C., Hjorth, J., \& Andersen, A. C. 2011b, A\&AR, 19, 43

[47] Rho, J., et al. 2009, ApJ, 700, 579 
[48] Sakon, I., et al. 2009, ApJ, 692, 546

[49] Maiolino, R., et al. 2004a, Nature, 431, 533

[50] Hirashita, H., Nozawa, T., Kozasa, T., Ishii, T. T., \& Takeuchi, T. T. 2005, MNRAS, 357, 1077

[51] Stratta, G., Maiolino, R., Fiore, F., \& D’Elia, V. 2007, ApJ, 661, L9

[52] Schady, P., et al. 2007, MNRAS, 377, 273

[53] Zafar, T., et al. 2011, A\&A, 532, A143

[54] Jang, M., Im, M., Lee, I., Urata, Y., Huang, K., Hirashita, H., Fan, X., \& Jiang, L. 2011, ApJ, 741, L20

[55] Perley, D. A., et al. 2010, MNRAS, 406, 2473

[56] Kawara, K., et al. 2010, MNRAS, 412, 1070

[57] Bianchi, S., \& Schneider, R. 2007, MNRAS, 378, 973

[58] Nozawa, T., et al. 2007, ApJ, 666, 955

[59] Hirashita, H., Nozawa, T., Takeuchi, T. T., \& Kozasa, T. 2008, MNRAS, 384, 1725

[60] Cardelli, J. A., Clayton, G. C., \& Mathis, J. S. 1989, ApJ, 345, 245

[61] Pei, Y. C. 1992, ApJ, 395, 130

[62] Valiante, R., Schneider, R., Bianchi, S., \& Andersen, A. C. 2009, MNRAS, 397, 1661

[63] Groenewegen, M. A. T. 1997, A\&A, 317, 503

[64] Gauger, A., Balega, Y. Y., Irrgang, P. Osterbart R., \& Weigelt G. 1999, A\&A, 346, 505

[65] Hofmann, K.-H., Balega, Y., Blöcker, T., \& Weigelt, G. 2001, A\&A, 379, 529-539

[66] Winters, J. M., Fleischer, A. J., Le Bertre, T., \& Sedlmayr, E. 1997, A\&A, 326, 305

[67] Yasuda, Y., \& Kozasa, T. 2012, ApJ, 745, 159

[68] Ventura, P., et al. 2012, MNRAS, 424, 2345

[69] Hoppe, P., Amari, S., Zinner, E., Ireland, T., \& Lewis, R. S. 1994, ApJ, 430, 870

[70] Daulton, T. L., et al. 2003, Geochim. Cosmochim. Acta, 67, 4743

[71] Amari, S., Lewis, R. S., \& Anders, E. 1994, Geochim. Cosmochim. Acta, 58, 459

[72] Hoppe, P., \& Zinner, E. 2000, J. Geophys. Res., 105, 10371

[73] Asano, R. S., Takeuchi, T. T., Hirashita, H., \& Nozawa, T. 2013a, MNRAS, 432, 637 
[74] Gallerani, S., et al. 2010, A\&A, 523, A85

[75] Hjorth, J., Vreeswijk, P. M., Gall, C., \& Watson, D. 2013, ApJ, 758, 173

[76] Maiolino, R., et al. 2004b, A\&A, 420, 889

[77] Dwek, E. 1987, ApJ, 322, 812

[78] Nozawa, T., Kozasa, T., \& Habe, A. 2006, ApJ, 648, 435

[79] Asano, R. S., Takeuchi, T. T., Hirashita, H., \& Nozawa, T. 2014, MNRAS, in press (arXiv:1401.7121)

[80] Andersen, M., Rho, J., Reach, W. T., Hewitt, J. W., \& Bernard, J. P. 2011, ApJ, 742, 7

[81] Seok, J. Y., Koo, B.-C., \& Onaka, T. 2012, ApJ, 744, 160

[82] Guillet, V., Pineau Des Forêts, G., \& Jones, A. P. 2011, A\&A, 527, A123

[83] McKee C. F., 1989, in Interstellar Dust, Proc. IAU Symp. 135, L. J. Allamandola \& A. G. G. M. Tielens eds., Kluwer, Dordrecht, p. 431

[84] Jones, A. P., \& Nuth, J. A. 2011, A\&A, 530, A44

[85] Serra Díaz-Cano, L., \& Jones, A. P. 2008, A\&A, 492, 127

[86] Mathis, J. S. 2000, in Circumstellar and Interstellar Material, Allen's Astrophysical Quantities, A. N. Cox ed., Springer, New York, p. 523

[87] Tielens, A. G. G. M. 2005, The Physics and Chemistry of the Interstellar Medium, Cambridge University Press, Cambridge

[88] Hirashita, H., Hibi, Y., \& Shibai, H. 2007, MNRAS, 379, 974

[89] Valiante, R., Schneider, R., Salvadori, S., \& Bianchi, S. 2011, MNRAS, 416, 1916

[90] Asano, R. S., Takeuchi, T. T., Hirashita, H., \& Inoue, A. K. 2013b, Earth Planets Space, 65, 213

[91] Bertoldi, F., et al. 2003, A\&A, 406, L55

[92] Robson, I., Priddey, R. S., Isaak, K. G., \& McMahon, R. G. 2004, MNRAS, 351, L29

[93] Priddey, R. S., Ivison, R. J., \& Isaak, K. G. 2008, MNRAS, 383, 289

[94] Michałowski, et al. 2010, A\&A, 522, A15

[95] Mattsson, L. 2011, MNRAS, 414, 781

[96] Dwek, E., Galliano, F., \& Jones, A. P. 2007, ApJ, 662, 927

[97] Kuo, T.-M., \& Hirashita, H. 2012, MNRAS, 424, L34 
[98] Inoue, A. K. 2011, Earth Planets Space, 63, 1027

[99] Hirashita, H. 2012, MNRAS, 422, 1263

[100] Hirashita, H., \& Voshchinnikov, N. V. 2014, MNRAS, 437, 1636

[101] Draine, B. T., \& Lee, H. M. 1984, ApJ, 285, 89

[102] Yan, H., Lazarian, A., \& Draine, B. 2004, ApJ, 616, 895

[103] Hirashita, H., \& Yan, H. 2009, MNRAS, 394, 1061

[104] Hirashita, H., Nozawa, T., Yan, H., \& Kozasa, T. 2010, MNRAS, 404, 1437

[105] Seok, J. Y., Hirashita, H., \& Asano, R. S. 2014, MNRAS, 439, 2186

[106] Jones, A. P. 2009, in Cosmic Dust - Near and Far, ASP Conference Series 414, Th. Henning, E. Grün, \& J. Steinacker eds., ASP, San Francisco, p. 473

[107] Galliano, F., Dwek, E., \& Chanial, P. 2008, ApJ, 672, 214

[108] Gordon, K. D., et al. 2008, ApJ, 682, 336

[109] Pagani, L., Steinacker, J., Bacmann, A., Stutz, A., \& Henning, T., 2010, Science, 329, 1622

[110] Steinacker, J., Pagani, L., Bacmann, L., \& Guieu, S., 2010, A\&A, 511, A9

[111] Ormel, C. W., Paszun, D., Dominik, C., \& Tielens, A. G. G. M. 2009, A\&A, 502, 845

[112] Hirashita, H., \& Li, Z.-Y. 2013, MNRAS, 434, L70

[113] Tinsley, B. M. 1980, Fundamentals of Cosmic Physics, 5, 287

[114] Zafar, T., \& Watson, D. 2013, A\&A, 560, A26

[115] Rémy-Ruyer, A., et al. 2014, A\&A, 563, A31

[116] Hirashita, H., \& Kuo, T.-M. 2011, MNRAS, 416, 1340

[117] Kobayashi, H., Tanaka, H., Krivov, A. V., \& Inaba, S. 2010, Icarus, 209, 836

[118] Hirashita, H., \& Nozawa, T. 2013, Earth Planets Space, 65, 183 